\title{
Mudança nos temas centrais do relacionamento conflituoso de pacientes ansiosos em psicoterapia psicodinâmica on-line
}

\author{
Change in the Core Conflictual Relationship Themes of anxious patients receiving online
} psychodynamic psychotherapy

Cambio en el Tema Central de Conflicto Relacional de pacientes ansiosos en psicoterapia psicodinámica on-line

\section{Resumo}

O relacionamento central conflituoso é um padrão pelo qual o indivíduo estabelece os seus relacionamentos interpessoais, sendo sua origem decorrente de conflitos centrais regressivos. Presume-se que, quanto mais rígidos os elementos do conflito, maior a severidade da psicopatologia. Ainda não se sabe se psicoterapias psicodinâmicas online produzem modificações em nível intrapsíquico e são capazes de alterar o padrão disfuncional dos pacientes. Portanto, objetivou-se explorar as eventuais mudanças no padrão de relacionamento conflitivo expressos nas sessões finais em relação às iniciais dos pacientes em psicoterapia psicodinâmica on-line. Trata-se de uma investigação naturalística e longitudinal por meio de estudo de caso sistemático. Participaram seis pacientes com sintomas de ansiedade. Utilizou-se o instrumento Core Conflictual Relationship Theme. Os dados foram coletados por meio da transcrição da gravação do áudio e vídeo das sessões que compuseram os tratamentos. Foram analisados, de forma descritiva e comparativa, 120 episódios de relacionamento em 25 sessões. Os resultados denotaram modificações nos desejos, respostas dos outros e de self que sinalizam mudança intrapsíquica no tratamento a distância. São apresentadas também evidências clínicas que podem guiar psicoterapeutas no processo de mudança psicoterápica. Limitações e sugestões para pesquisas futuras são apresentadas.

Palavras-chave: Psicoterapia psicodinâmica; Transtornos de ansiedade; Resultados terapêuticos; Psicoterapia on-line.

\begin{abstract}
The core conflictual relationship is a pattern by which the individual establishes their interpersonal relationships, and its origin derives from regressive core conflicts. It is not known whether online psychodynamic psychotherapies produce changes at the intrapsychic level and are capable of altering the dysfunctional pattern of patients. Therefore, the aim was to explore possible changes in the conflictual relationship pattern expressed in the final sessions in relation to the initials sessions of patients receiving online psychodynamic psychotherapy. This was a naturalistic and longitudinal investigation through a systematic case study. Six patients with symptoms of anxiety participated. The Core Conflictual Relationship Theme instrument was used to extract the central relational pattern of the patients from
\end{abstract}


the psychotherapy narratives. The data were collected through the transcription of the audio and video recordings of the sessions that comprised the treatments. A total of 120 relationship episodes, from 25 sessions, were descriptively and comparatively analyzed. The results showed changes in desires, and responses of others and self that indicate intrapsychic changes due to the distance treatment. Clinical evidence is also presented that can guide psychotherapists in the process of psychotherapeutic change. Limitations and suggestions for future research are discussed.

Keywords: Psychodynamic psychotherapy; Anxiety disorders; Therapeutic outcome; On-line psychotherapy.

\section{Resumen}

La relación central conflictiva es un patrón mediante el cual el individuo establece sus relaciones interpersonales, siendo su origen debido a los conflictos centrales regresivos. Se supone que cuanto más rígidos sean los elementos del conflicto, mayor será la gravedad de la psicopatología. Aún no se sabe si las psicoterapias psicodinámicas online son capaces de alterar el patrón disfuncional de los pacientes. Por lo tanto, el objetivo fue explorar los posibles cambios en el patrón de relación conflictivo expresado en las sesiones finales en relación con las iniciales de los pacientes en psicoterapia psicodinámica on-line. Es una investigación naturalista y longitudinal a través de un estudio de caso sistemático. Participaron seis pacientes con síntomas de ansiedad. Se utilizó el instrumento Core Conflictual Relationship Theme. Los datos fueron recolectados mediante la transcripción de las grabaciones de audio y video de las sesiones que componían los tratamientos. Analizamos, de forma descriptiva y comparativa, 120 episodios de relación en 25 sesiones. Los resultados denotaron cambios en los deseos, las respuestas del objeto y respuestas del sujeto que señalan un cambio emocional en el tratamiento on-line. También se presentan evidencias clínicas que pueden orientar a los psicoterapeutas en el proceso de cambio psicoterapéutico. Se presentan limitaciones y sugerencias para futuras investigaciones.

Palabras clave: Psicoterapia psicodinámica; Trastorno de ansiedad; Resultados terapéuticos; Psicoterapia on-line.

\section{Introdução}

Há evidências de que a psicoterapia on-line funciona para diminuir os sintomas dos pacientes em distintas condições clínicas (Barak et al., 2008; Rees et al., 2015; Varker et al., 2018). Dentre os estudos que indicam tais benefícios, grande parte estão orientados por abordagens cognitiva-comportamentais ou psicoeducativas (Feijó et al., 2018). Logo, existe a necessidade de se estudar a efetividade da psicoterapia na abordagem dinâmica, não somente em relação aos sintomas, mas também em relação às mudanças estruturais intrapsíquicas (Beutel et al., 2018), uma vez que se reconhece que um dos efeitos esperados nas psicoterapias psicanalíticas seria a redução dos sintomas e dos problemas interpessoais dos pacientes (Castro, 2020; Gaston et al., 1994; Wilczek et al., 2004).

Com o intuito de sistematizar um modelo que capte os problemas interpessoais dos pacientes e a compreensão de conteúdos emocionais, interpessoais e repetitivos que se apresentam nas sessões de psicoterapia, Luborsky e colaboradores sistematizaram o Core Conflictual Relationship Theme, que em português é denominado de "Método do Relacionamento Central Conflituoso", com acrônimo de CCRT (Luborsky et al., 1994). O instrumento possibilita compreender a conflitiva intrapsíquica por meio de padrões ou temas centrais que se assemelham ao conceito de padrão transferencial presente nas obras Freudianas (Barrett, 2009; Rocha, 2013), sendo considerado uma padronização deste construto (Luborsky, 1984).

Desenvolvido na década de 70 como método de formulação de caso e de pesquisa em psicoterapia, o CCRT permite compreender como que cada pessoa se relaciona com outras pessoas, analisando os episódios de relacionamento (ERs) que são narrados na sessão. Supõe-se que formas repetitivas de se relacionar apresentam elementos emocionais marcantes que estiveram presentes nos relacionamentos com as figuras de cuidado e que são reatualizadas nos relacionamentos subsequentes, sendo ativados com maior frequência na relação com o terapeuta, isto é, transferencialmente (Luborsky, 1984; Rocha, 2013).

Entende-se que nas situações interpessoais significativas a pessoa expressa desejos, necessidades e intenções dirigidas a alguém significativo e, a partir disso, esta pessoa reage a essas expectativas de forma positiva ou negativa, gratificando ou frustrando os desejos. Como consequência, o desejante manifesta implicitamente ou explicitamente uma reação emocional em função da forma com que os outros reagiram às suas intenções (Rocha, 2013). Essa triangulação expressa os episódios de relacionamento em três domínios, os desejos (D), as Respostas dos Outros (RO) e a Resposta de Selfleu (RS ou RE) do 
paciente com terceiros, com o próprio paciente ou com o terapeuta (Luborsky et al., 1994).

Diversos estudos utilizaram o CCRT para compreender o padrão de relacionamento central e a qualidade das interações interpessoais em diversas situações cínicas e psicopatológicas (Barrett, 2009; Beutel et al., 2018; Bottino et al., 2003; Castro, 2020; Crits-Christoph et al., 1996, 2004; Duarte et al., 2001; Salgado \& Pires, 2014; Silva \& Serralta, 2013). A utilização desse método, que já é difundido em diversas pesquisas em psicoterapia psicodinâmica, é possivelmente um dos melhores recursos sistematizados existentes para examinar a dinâmica relacional dos pacientes.

Além disto, o método pode servir como uma importante ferramenta na investigação empírica da psicoterapia psicodinâmica, uma vez que a qualidade das interações interpessoais pode indicar como o paciente responde ao trabalho psicoterapêutico e ainda avaliar a mudança intrapsíquica. Assim, o método CCRT oportuniza uma análise formal de um tratamento, pois às vezes as avaliações são realizadas informalmente pelos psicoterapeutas (Santos, 2013).

O CCRT já foi utilizado para identificar o padrão central conflitivo de pacientes com transtornos de ansiedade na psicoterapia psicodinâmica presencial, por Duarte et. al. (2001). Na formulação de caso dos 15 pacientes avaliados, realizada no início do tratamento, encontrou-se como desejo mais frequente o de ser controlado, ser ferido e não ser responsável (em $40 \%$ dos casos), seguido de ser amado e compreendido (33,3\% dos entrevistados). Enquanto respostas dos outros, observou-se a dominância de respostas de rejeição e oposição $(73,3 \%)$, seguidos de fora de si $(13,3 \%)$. Por último, o domino resposta do self resultou como desapontados e deprimidos $(86,7 \%)$.

Um outro estudo em psicoterapia presencial, sobre padrões de relacionamento interpessoais recorrentes no transtorno de ansiedade generalizada apontou que, como formulação de caso, esses pacientes desejavam ser amados e compreendidos, percebiam os outros como pessoas não ajudantes e opositoras e apresentavam resposta do self de desamparo, falta de amor e ansiedade (Crits-Christoph et al., 2004). Ademais, um estudo de caso sistemático de uma paciente com sintomas de ansiedade e depressão evidenciou o padrão conflitivo central dela sendo, o desejo de sentir-se cômoda, as resposta dos outros de cooperação coexistentes com as de fortes e resposta de self de comodidade simultâneas com as de não receptiva e ansiosa (Silva \& Serralta, 2013).

No contexto on-line assíncrono, a formulação de caso com o CCRT foi utilizada em dois tratamentos conduzidos por escrito e orientados pela terapia suportiva-expressiva de Luborsky. Os autores concluíram que ambos os processos psicoterápicos foram exitosos e que as formulações contribuíram para modificar as interações interpessoais desadaptativas por meio de intervenções terapêuticas expressivas e de apoio (Beutel et al., 2018; Zwerenz et al., 2017). Ainda que esses estudos sugiram os efeitos das intervenções a distância sobre estrutura e dinâmica intrapsíquica, estas evidências precisam ser replicadas e expandidas. É digno de nota que, até o momento, não foram identificados estudos que avaliaram a descrição, resultado ou nível da mudança intrapsíquica no contexto psicoterápico on-line síncrono.

Quando o paciente busca auxílio em uma psicoterapia psicodinâmica, mesmo que nas intervenções on-line, o alívio dos sintomas e as dificuldades de relacionamento interpessoal poderiam ser temas importantes para compor os objetivos do tratamento em uma perspectiva psicodinâmica relacional (Barrett, 2009; Castro, 2020; Salgado \& Pires, 2014), pois a forma com que as pessoas narram os seus relacionamentos com outros indivíduos, somadas aos seus traços de personalidade subjazem a manifestação/presença de sintomas psicopatológicos. Entende-se que, por meio da melhora na qualidade das narrativas nestes relacionamentos, além da redução dos sintomas, poder-se-ia denotar o progresso psicológico do paciente (Barrett, 2009; Castro, 2020).

O objetivo deste estudo foi o de apresentar uma descrição do CCRT comum aos casos em análise e avaliar as possíveis mudanças no padrão de relacionamento conflitivo dos pacientes em termos de desejos, respostas do self e resposta dos outros expressos nas sessões finais do tratamento em relação às sessões iniciais em psicoterapias psicodinâmicas on-line. 
Desse modo, com o estudo pretende-se contribuir com a avaliação da extensão das mudanças que podem ser obtidas nesta modalidade de psicoterapia e, para difundir entre clínicos e pesquisadores, o método CCRT.

\section{Metodologia}

\section{Delineamento}

Utilizou-se do delineamento longitudinal, naturalístico, com base no estudo de caso sistemático - ECS (Edwards, 2007). O ECS é utilizado nas pesquisas de processos psicoterápicos com o intuito de garantir melhor aprimoramento metodológico, uma vez que utiliza material clínico gravado em áudio e/ou vídeo, e utiliza procedimentos sistemáticos para análise de dados, via de regra, feita por juízes de forma independente (Serralta et al., 2011).

\section{Os casos em análise}

O presente estudo é derivado de um projeto mais amplo sobre processo-resultado da psicoterapia psicodinâmica online de autoria do primeiro autor. Os participantes foram escolhidos por conveniência em uma divulgação da pesquisa por mídias sociais (Creswell, 2010), sendo seis mulheres que foram atendidas em psicoterapia psicodinâmica à distância, por videoconferência, por três psicoterapeutas, psicólogas, do sexo feminino, que possuíam treinamento em psicoterapia on-line e especialização em psicoterapia psicanalítica.

Em relação as pacientes, elas preencheram um questionário on-line e depois de elegíveis, conforme preenchimento do questionário e do Termo de consentimento Livre e Esclarecido, foram encaminhadas à triagem à distância, por videoconferência, quando realizaram uma entrevista com o primeiro autor para avaliação técnica do atendimento, verificação do preenchimento dos critérios clínicos para inclusão, sendo maiores de idade, com queixa circunscrita relacionada à ansiedade, com sintomas em nível ao menos moderado, e sem sintomas psicóticos ou outras condições graves que poderiam não indicar o tratamento. Após avaliadas, elas foram encaminhadas às terapeutas para o início do tratamento.

As participantes designadas como pacientes são do sexo feminino, de cor branca, moradoras no sul do Brasil, quatros delas com ensino superior completo e duas cursando bacharelado. Possuíam entre 22 e 37 anos $\left({ }^{\bar{x}}=29,33\right.$ anos; dp $=5,43$ anos) e apresentavam queixas de ansiedade. Além disto para participarem do tratamento, precisavam pontuar pelo menos 10 pontos na Generalized Anxiety Disorder 7-item (GAD-7), o que corresponde ao nível moderado de intensidade de sintomas (Spitzer et al., 2006).

Por fim, nenhuma fez uso de medicação psiquiátrica no decorrer do processo psicoterápico. Além disto, consideravam-se usuárias avançadas da internet. Na Tabela 1 apresentam-se as formulações psicodinâmicas das psicoterapeutas sobre os casos em análise. 
Research, Society and Development, v. 11, n. 2, e41711225918, 2022

(CC BY 4.0) | ISSN 2525-3409 | DOI: http://dx.doi.org/10.33448/rsd-v11i2.25918

Tabela 1 - Formulações Psicodinâmicas dos casos elaboradas pelas psicoterapeutas

\begin{tabular}{|c|c|}
\hline Nome & Formulação Psicodinâmica \\
\hline Alessandra & $\begin{array}{l}\text { Paciente buscou atendimento por sentir-se ansiosa e perdida frente as atividades da pós-graduação. Apresenta } \\
\text { relacionamentos próximos e estáveis, mas algumas dificuldades no âmbito das interações familiares. Apresenta } \\
\text { conflito em ser independente. Possui características neuróticas de funcionamento que incluem necessidade de } \\
\text { cumprir tarefas com excelência, agradar e obter aprovação de outros, dificuldade para lidar com frustações e } \\
\text { imperfeições, e uso de defesas de intelectualização para controlar a ansiedade. Paciente apresenta doenças } \\
\text { orgânicas que reforçam sua necessidade de ser cuidada. }\end{array}$ \\
\hline Daiana & $\begin{array}{l}\text { Paciente buscou atendimento por insegurança, angústia e dificuldade na criação dos filhos. Sente-se desamparada } \\
\text { e sobrecarregada com a maternidade. Possui conflito com a própria mãe, sentindo-se julgada e cobrada por ela. }\end{array}$ \\
\hline Paula & $\begin{array}{l}\text { Paciente buscou atendimento por apresentar ansiedade relacionada à mudança de curso de graduação e } \\
\text { amadurecimento. Apresenta alto nível de cobrança em relação a si mesma, deseja crescer e ser potente ao mesmo } \\
\text { tempo em que não se permite envolver verdadeiramente nas atividades que proporcionariam esse crescimento. } \\
\text { Apresenta características de personalidade neuróticas do tipo predominantemente obsessivo, usando } \\
\text { primordialmente os mecanismos de defesa de idealização e desvalorização, formação reativa e isolamento entre } \\
\text { ideia e afeto como modo de controlar a ansiedade. }\end{array}$ \\
\hline Renata & $\begin{array}{l}\text { Paciente buscou atendimento devido à sintomas somáticos de ansiedade, de depressão e somatização em resposta } \\
\text { às situações estressantes, especialmente no trabalho. Introvertida, apresenta baixa autoestima. Refere que sua } \\
\text { família apresenta relações conflituosas e fragilidades com as quais não sabe como lidar, sentindo-se desamparada } \\
\text { e impotente. Apresenta dificuldade em expressar por palavras suas angústias e, quando se sente vulnerável, } \\
\text { espera que alguém a defenda. }\end{array}$ \\
\hline Taila & $\begin{array}{l}\text { Paciente buscou atendimento devido a ansiedade frente o término da graduação. Possui histórico de sintoma } \\
\text { alimentar tratado com sucesso na etapa inicial da adolescência. Paciente sensível a críticas, necessidade de } \\
\text { agradar as pessoas e insegurança frente a novas relações sociais. Teme situações de exposição, o que desencadeia } \\
\text { necessidade de constante controle das situações. }\end{array}$ \\
\hline Valéria & $\begin{array}{l}\text { Paciente buscou atendimento com queixas de ansiedade relacionada ao término de bolsa de doutorado e incerteza } \\
\text { em relação ao futuro profissional. É auto exigente, tímida e teme ser julgada por figuras de autoridade. Utiliza } \\
\text { defesas obsessivas para lidar com a ansiedade. Busca aprovação e necessidade de constante apoio. }\end{array}$ \\
\hline
\end{tabular}

Fonte: Autores.

As psicólogas foram selecionadas para a pesquisa por serem integrantes da rede de contato do primeiro autor e por estarem vinculada ao grupo de pesquisa dos autores. Pacientes e terapeutas aceitaram participar da pesquisa e assinaram o Termo de Consentimento Livre e Esclarecido. As terapeutas possuíam entre 30 e 60 anos $\left({ }^{\bar{X}}=43,33\right.$ anos; dp = 15,28 anos) e experiência clínica de 4 a $25 \operatorname{anos}\left(^{\bar{X}}=15,33\right.$ anos; dp $=10,60$ anos). O tempo médio da conclusão da formação em psicoterapia psicodinâmica foi de 7 anos.

\section{Tratamento}

Em relação ao tratamento, foram ofertados a cada uma das pacientes um total de 24 sessões de psicoterapia, com frequência semanal, por videoconferência, de forma gratuita, utilizando a Plataforma de saúde mental da empresa Vittude ${ }^{\circledR}$ para o consultório virtual. Todos os tratamentos foram conduzidos de forma naturalística, sendo 5 deles bem-sucedidos em 
termos de mudanças sintomáticas avaliado pelo GAD-7 (Spitzer et al., 2006) e no bem estar psicológico pelo Clinical Outcome Routine Evaluation - Outcome Measure (Evans et al., 2002), considerando o pré e pós-tratamento e no follow-up, realizado aos três meses. Todas as sessões foram gravadas em áudio e vídeo por meio de aplicativo instalado no computador da terapeuta.

Para as pacientes foi ofertado a Psicoterapia Psicodinâmica de abordagem relacional. Ela centra-se na investigação do afeto e na manifestação da emoção, na identificação de temas e padrões repetitivos, na discussão de experiências ao longo do desenvolvimento, apresenta foco nos relacionamentos interpessoais e terapêutico e na vida em fantasia, utilizando-se da autorreflexão e do autoexame, como forma de entender os padrões de relacionamento problemáticos do paciente (Shedler, 2010). A abordagem relacional demarca o paradigma psicanalítico contemporâneo que considera os relacionamentos, internalizados ou não, como centrais à compreensão do comportamento do paciente e à intervenção terapêutica (Malone, 2018).

\section{Instrumentos}

Ficha de dados sociodemográficos. Instrumento elaborado pelo autor para coleta de dados relacionados com a idade, gênero, cor da pele, estado civil, escolaridade, situação ocupacional, contato pessoal, região geográfica de moradia e usabilidade das tecnologias de informação e comunicação.

Generalized Anxiety Disorder (Spitzer, Kroenke, Williams, \& Löwe, 2006). É um instrumento de autorrelato, com sete itens, sensível ao Transtorno de Ansiedade Generalizada, como também para o Transtorno do Pânico, Transtorno de Ansiedade Social e Transtorno de Estresse Pós-Traumático (Spitzer et al., 2006). No Brasil, a escala foi denominada Questionário de Transtorno de Ansiedade Generalizada (Moreno et al., 2016) e apresentou coeficiente de Alfa foi de $\alpha=0,916$ e o rho coeficiente de confiabilidade composta de $\rho=0,909$, com a população universitária. O ponto de corte, com relação ao sexo, na escala é de 9,69 para a população feminina (Moreno et al., 2016).

Core Conflictual Relationship Theme Method (Luborsky et al., 1994). Instrumento traduzido no Brasil como Método do Tema Central do Relacionamento Conflituoso (Yoshida et al., 2009). Sua aplicação resulta na identificação do padrão central que cada indivíduo estabelece nos seus relacionamentos interpessoais (Rocha, 2013). Para a sua extração, é necessária a leitura integral das transcrições das sessões de psicoterapia e a identificação dos trechos em que há um episódio relacional em ocorrência. Esses episódios de relacionamento (ERs) extraídos das transcrições são grifados e codificados pelo leitor em domínios de desejo (D), Resposta do Self (RS) e Resposta do Outro (RO), estes dois últimos sendo divididos em positivos ou negativos (em termos de gratificação ou frustração).

Os domínios do CCRT podem ser categorizados de forma personalizada e idiossincrática para cada pessoa, que é chamando de tailor-made, ou operacionalizado por meio de categorias, ou cluster, previamente definidas em pesquisas e que constam no manual - atualmente em sua terceira edição (Luborsky et al., 1994; Rocha, 2013). Na terceira edição há oito categorias, cluster, para cada domínio - desejo, respostas dos Outros e repostas de self.

Para o episódio de relacionamento ser válido e passível de consideração para a formulação final do CCRT, cada um deles deve apresentar completude mínima de 2,5 pontos em uma escala de zero a cinco pontos (Luborsky et al., 1994). Ademais, são identificadas as pessoas significativas mais frequentes nos ERs e o tempo em que a interação ocorreu, sendo passado - há mais de três anos; recente em até três anos e atual, sendo dentro da sessão ou até poucas semanas. Para concluir um padrão de relacionamento conflitivo, faz-se necessário pelo menos 10 ERs implícitos ou explícitos nas narrativas de cada caso. Quando o CCRT é utilizado para avaliar o resultado de uma intervenção psicoterápica, faz-se necessária a extração destes episódios em cada etapa ou seguimento do tratamento. 


\section{Procedimento de coleta de dados}

Neste estudo os dados foram coletados por meio das psicoterapias psicodinâmicas realizadas on-line e gravadas em áudio e vídeo que compuseram o estudo de doutorado do primeiro autor. Esses dados foram gravados utilizando Software Movavi Screen Recorder. Posteriormente as gravações foram transcritas literalmente por bolsistas de iniciação científica vinculados ao grupo de pesquisa.

\section{Procedimento de análise de dados}

Uma vez que o procedimento de codificação do CCRT necessita de pelo menos 10 episódios de relacionamento completos (Luborsky et al., 1994), foram analisadas as sessões necessárias de cada etapa (inicial e final) de cada caso para cumprir esse critério. Em cada caso, foi necessário entre uma e três sessões de cada período (inicial e final). Portanto, compuseram o corpus de análise 15 sessões do início dos tratamentos e 10 sessões do seguimento final, totalizando 25 sessões examinadas. O processo de codificação ocorreu utilizando uma equipe composta de quatro bolsistas de iniciação científica coordenados pelo primeiro autor do estudo (doutorando), todos já treinados no uso do método e com experiência em pesquisas anteriores.

Inicialmente, foi realizada a transcrição dos vídeos das sessões de psicoterapia pelos bolsistas de iniciação científica. A formulação do CCRT foi baseada em duas fases: localização dos episódios de relacionamento (ERs) e identificação dos componentes Desejo (D), Resposta do Outro (RO) e Resposta do Self (RS), como descrito a seguir.

Os ERs foram numerados por ordem de ocorrência nas sessões de psicoterapia transcritas. Neles, identificou-se o nome, o tipo de relação estabelecida (pai, mãe, filhos, chefia, esposa, marido, entre outros), a data em que o ER ocorreu sendo atual, recente ou passado e a idade do paciente na ocasião do episódio. Para cada ER localizado, foi atribuído um escore que variou em uma escala likert de 1 a 5 pontos. Somente foram avaliados os ERs que receberam notas superiores a 2,5 pontos na avaliação de dois juízes independentes.

Depois, ocorreu a etapa de identificação dos Ds, ROs e REs em cada ERs, sendo que os dois últimos domínios ainda foram classificados em positivos ou negativos. Posteriormente, descreveu-se e quantificou-se os Ds, ROs e Rs da sessão e por etapa do tratamento, ao final formulou-se a frequência destas classificações para a versão inicial e final do CCRT de cada paciente. Essas etapas também foram conduzidas por duplas de juízes independentes, sendo alunos de graduação e pósgraduação treinados e com experiência no instrumento. Nos momentos de discrepâncias entre os juízes, foi utilizado o consenso entre ambos.

Neste estudo, foram considerados para avaliação os clusters da $3^{\text {a }} \mathrm{e}$ atual edição do CCRT. No conjunto dos casos, foi realizada uma análise descritiva, comparativa e de frequência entre o início e final do tratamento por meio do Software Statistical Package for the Social Sciences.

\section{Procedimentos éticos}

A pesquisa foi aprovada pelo Comitê de Ética em Pesquisa da Universidade do Vale do Rio dos Sinos (CAAE 09821919.5.0000.5344).

\section{Resultados}

Cinco dos seis casos em análise apresentaram mudança sintomatológica compatível com a melhora clinicamente significativa, que foi verificado pelo Reliable Change Index (Jacobson \& Truax, 1991), tais resultados são descritos na Tabela 
Research, Society and Development, v. 11, n. 2, e41711225918, 2022

(CC BY 4.0) | ISSN 2525-3409 | DOI: http://dx.doi.org/10.33448/rsd-v11i2.25918

Tabela 2 - Mudança clinicamente significativa nos sintomas de ansiedade (GAD-7)

\begin{tabular}{llllll}
\hline Paciente & Escore & Escore & RCI & Escore & RCI \\
& Pré-Tratamento & Pós-Tratamento & Pré/Pós-tratamento & Follow-up & Pré - Tratamento e Follow-up \\
Alessandra & 13 & 12 & 0,44 & - & - \\
Daiana & 10 & 1 & $3,92^{*}$ & 2 & $3,49^{*}$ \\
Paula & 16 & 6 & $4,36^{*}$ & 4 & $5,23^{*}$ \\
Renata & 12 & 7 & $2,18^{*}$ & 3 & $3,93^{*}$ \\
Taila & 15 & 4 & $4,79^{*}$ & 6 & $3,92^{*}$ \\
Valeria & 11 & 1 & $4,36^{*}$ & 1 & $4,36^{*}$ \\
\hline
\end{tabular}

Nota. $*$ Mudança clinicamente significativa. Fonte: Autores.

Em relação a mudança no relacionamento conflituoso, no conjunto de narrativas das participantes, foram analisados 120 episódios de relacionamento, sendo 60 do início de tratamento e 60 do seguimento final, correspondendo aos 10 primeiros e 10 finais ERs completos de cada caso. A Figura 1 apresenta a frequência dos componentes do CCRT na soma dos casos da pesquisa, ponderando o início e o final do processo psicoterapêutico. 
Research, Society and Development, v. 11, n. 2, e41711225918, 2022

(CC BY 4.0) | ISSN 2525-3409 | DOI: http://dx.doi.org/10.33448/rsd-v11i2.25918

Figura 1. Descrição comparativa dos Desejos, Respostas dos Outros e Respostas do Self no início e no final do tratamento.

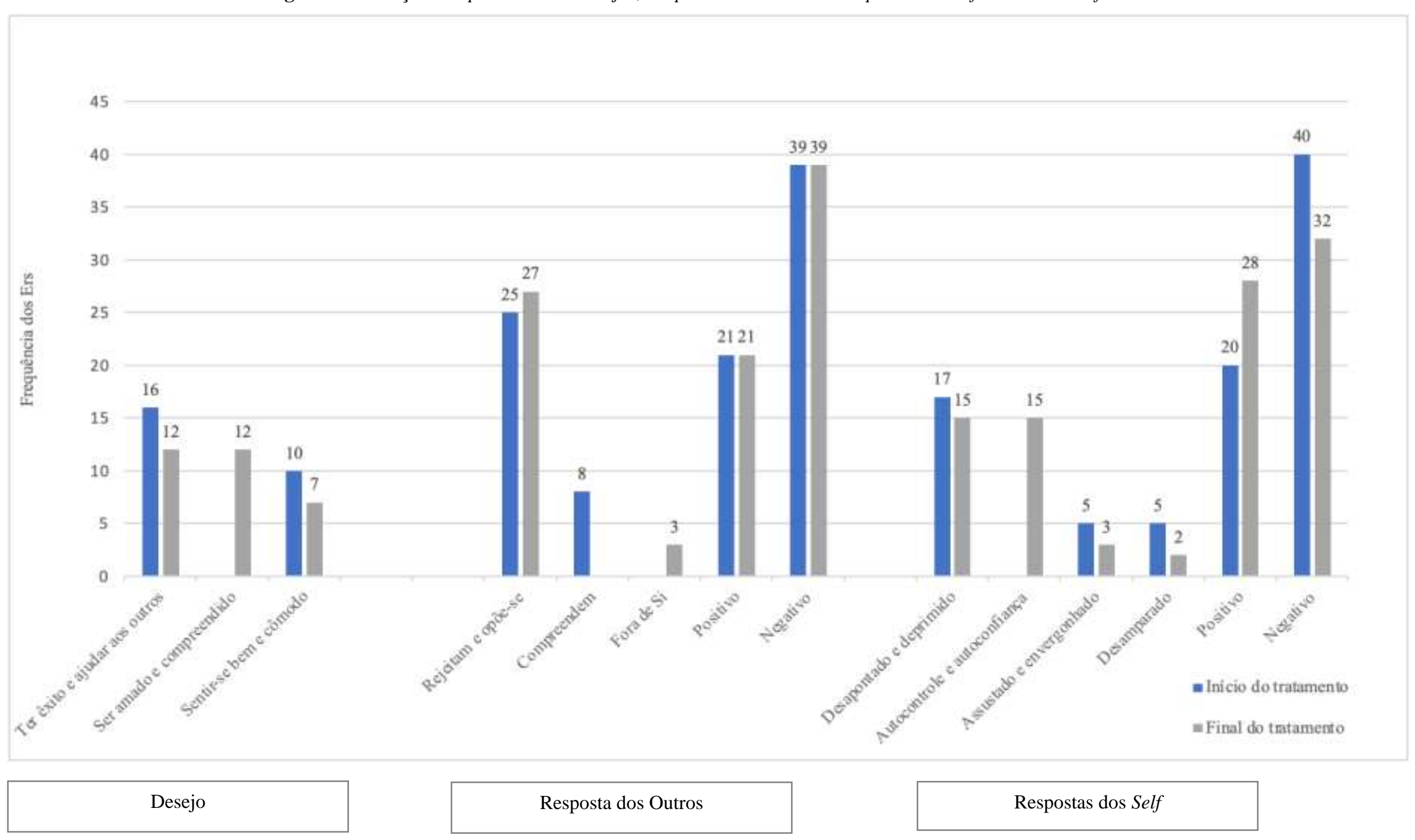

Fonte: Autores. 
Levando em conta as seis pacientes, na etapa inicial encontrou-se como desejo principal o de "ter êxito e ajudar os outros" (26,7\%), seguido do desejo secundário de "sentir-se bem e cômodo" (16,7\%). No período do final do tratamento, verificou-se que os desejos primários se modificaram, pois "ter êxito e ajudar os outros" permaneceu inalterado (20\%), "ser amado e compreendido" tornou-se primário (20\%) e "sentir-se bem e cômodo" como secundário (11,7\%).

A direção da resposta dos outros na etapa inicial foi negativa (65\%), ou seja, relacionada à frustração, sendo a resposta mais frequente, de que os outros "rejeitam e opõem-se" $(41,7 \%)$ e, em menor grau, "compreendem" (13,3\%). Na etapa final, a direção da resposta do outro também se manteve negativa (65\%), assim como no período inicial. A resposta dos outros de maior frequência foi "rejeitam e opõem-se" (45\%), tal como observado no período inicial do tratamento. A segunda mais frequente foi a resposta "fora de si - fora de controle" (5\%) que se apresentou somente nesta etapa do tratamento.

Em relação à resposta de self da etapa inicial, a maioria foi negativa $(66,7 \%)$, sendo que as respostas mais prevalentes foram "desapontado e deprimido" (28,3\%), "desamparado" (8,3\%) e "assustado e envergonhado" (8,3\%). Tendo em vista a resposta de self no seguimento final, há um decréscimo nas respostas negativas, mas seguem sendo a maioria $(53,3 \%)$. Entretanto, entre as primárias mais prevalentes, “desapontado e deprimido", embora tenha diminuído, se manteve no período final (no final em 25\%) enquanto outra, autocontrole e autoconfiança, passou a compor as primárias na etapa final (25\%). Já entre as respostas do self secundárias, a categoria "assustado e envergonhado" diminuiu (no final em 5\%).

Por fim, observou-se que na etapa inicial as pessoas mais frequentes nos episódios conflitivos relatados eram provenientes do ambiente de trabalho (38,3\%), seguido por pessoas da família (35\%). Já no período final, as pessoas mais frequentes nos ERs ativados foram as oriundas do ambiente familiar $(43,3 \%)$ existindo, portanto, uma modificação em relação ao período inicial. No tocante ao tempo dos episódios, no período inicial o mais prevalente foi classificado como recente, ou seja, as situações relatadas ocorreram nos últimos 3 anos (50\%). Na etapa final, esteve majoritariamente relacionado ao período atual (95\%), assim como ocorreu na etapa inicial do tratamento. A Tabela 3 sintetiza o CCRT comparativo entre o período inicial e final de tratamento no conjunto dos casos, sendo que em negrito marcou-se a diferença do final para o início.

Tabela 3 - CCRT inicial e final no conjunto dos casos.

\begin{tabular}{|c|c|c|c|}
\hline $\begin{array}{l}\text { Etapa do } \\
\text { tratamento }\end{array}$ & Desejo & Resposta do Outro & Resposta do Self \\
\hline & $1^{\circ}$. Ter êxito e ajudar os outros & $1^{\circ}$. Rejeição e oposição & $1^{\circ}$. Desapontado e deprimido \\
\hline Início & $2^{\circ}$. Sentir-se bem e cômodo & $2^{\circ}$. Compreensão & $\begin{array}{l}2^{\circ} \text {. Desamparado } \\
2^{\circ} \text {. Assustado e envergonhado }\end{array}$ \\
\hline Final & $\begin{array}{l}1^{\circ} \text {. Ter êxito e ajudar os outros } \\
\mathbf{1}^{\circ} \text {. Ser amado e compreendido } \\
2^{\circ} \text {. Sentir-se bem e cômodo }\end{array}$ & $\begin{array}{l}1^{\circ} . \text { Rejeitam e opõem-se } \\
2^{\circ} . \text { Fora de si (fora de } \\
\text { controle) }\end{array}$ & $\begin{array}{l}1^{\circ} \text {. Desapontado e deprimido } \\
\mathbf{1}^{\mathrm{o}} \text {. Autocontrole e autoconfiança } \\
2^{\mathrm{o}} \text {. Assustado e envergonhado }\end{array}$ \\
\hline
\end{tabular}

Nota. Tabela elaborada pelos autores. Clusters demarcados em negrito representam a mudança observada nos componentes entre o período final se comparado ao inicial. Fonte: Autores.

\section{Discussão}

Compreender a extensão das mudanças estruturais nos relacionamentos conflituosos em intervenções psicodinâmicas on-line é pertinente, pois contribui para examinar se os tratamentos a distância promovem mudanças semelhantes às presenciais. Neste estudo, pode-se observar que as alterações nos relacionamentos centrais conflituosos das pacientes entre o início e o final dos atendimentos são compatíveis com mudanças intrapsíquicas. 
Com base no método CCRT, foi possível identificar um padrão comum às pacientes. Nesta pesquisa, os desejos expressos pelas pacientes, como o de ser amado e compreendido, foram identificados também em outros estudos com pacientes ansiosos (Crits-Christoph et al., 2004; Duarte et al., 2001), assim como a necessidade de se sentir bem e cômodo (Silva \& Serralta, 2013). A mudança nos desejos ao final de uma psicoterapia não é uma ocorrência comum, pois eles tendem a persistir, uma vez que essa seria uma característica idiossincrática do paciente (Castro, 2020).

No conjunto dos casos de psicoterapia on-line, a mudança em parte dos desejos foi denotada ao final do tratamento, entretanto, nos estudos com narrativas relacionais que compararam início e final de tratamentos presenciais, se constata que seria mais frequente a manutenção dos desejos, mesmo quando há progresso/benefícios no tratamento e que as mudanças ocorreriam somente nas respostas dos outros e de self (Castro, 2020; Rosbrow, 1995). Presume-se que a mudança incide mais sobre as respostas ou reações do que sobre os desejos, sendo isso suficiente para que se modifique toda a dinâmica relacional do indivíduo.

Em relação às respostas dos outros, predominaram as negativas, como as de rejeição e oposição. Ao final do tratamento, se mantiveram mais frequentes as negativas, porém mais fracas, ao mesmo tempo em que emergiram respostas indicativas de que as pacientes passaram com alguma frequência a avaliar as respostas dos outros como fora de si. A resposta de rejeição e oposição também são encontradas em outros estudos com pacientes ansiosos em psicoterapia presencial (CritsChristoph et al., 2004; Duarte et al., 2001; Silva \& Serralta, 2013), tal como notou-se em relação aos desejos. Isso pode sugerir a existência de um conflito comum subjacente aos sintomas ansiosos de pacientes supostamente neuróticos (Crits-Christoph et al., 2004).

Tais resultados evidenciados também podem denotar relação com o apego inseguro, quando a ambivalência na resposta das figuras de referência é significativa e repercute em sintomas emocionais de desamparo, abandono e falta de confiança (Benetti et al., 2018; Crits-Christoph et al., 2004). Já as respostas do domínio fora de si, ao final do tratamento, poderiam estar associadas a um conteúdo projetivo que, neste caso, pode ter efeito protetivo (defensivo) em relação à ansiedade; ou seja, no início do tratamento a percepção das pacientes parece indicar maior vulnerabilidade da autoestima, enquanto ao final elas supostamente passaram a compreender que o outro apresenta limitações externas as pacientes. Essa hipótese é coerente com o resultado de que ao final do tratamento há a manifestação de respostas do self positivas, como revela o autocontrole e autoconfiança, que coexistem com as respostas negativas já observadas no início da psicoterapia.

Ademais, respostas como as de desamparo, ansiedade, desapontamento e depressão fizeram-se também presentes em outros estudos em pacientes com ansiedade (Crits-Christoph et al., 2004; Duarte et al., 2001; Silva \& Serralta, 2013). A sua presença no final do tratamento de pacientes que tiveram mudanças sintomáticas significativas revela que há elementos do conflito subjacente que não se esgotam, porém amenizam e flexibilizam.

Chama atenção a frequência das respostas negativas do self, que, ainda que estivessem em maior número também ao final do tratamento, diminuíram se comparadas ao início do processo. Além disto, observou-se também um maior número de respostas positivas. Esse resultado pode indicar que com o tratamento on-line ofertado, as pacientes poderiam ter se beneficiado de certa flexibilidade e expansão da capacidade mental, assim como diminuição dos sintomas e inibições (Castro, 2020; López Moreno et al., 2005; Luborsky \& Crits-Christoph, 1990; Wilczek et al., 2004), uma vez que há o aumento de respostas positivas de self e um leque maior de reações.

Em relação à frequência das pessoas significativas no relacionamento conflitivo destas pacientes, observou-se que no início do tratamento as pessoas mais citadas eram do ambiente laboral e ao final as pessoas da família. É sabido que as relações pessoais no trabalho e as atividades laborais são importantes na vida das pessoas (Sturza \& Marques, 2017) e que podem favorecer o adoecimento mental (Fernandes et al., 2018), o que reforça a necessidade de incluir esses elementos quando se 
planeja ou avalia os objetivos terapêuticos de um paciente.

Deve-se ponderar que a diminuição nas narrativas das pacientes sobre os episódios recentes e o aumento das narrações de situações atuais ao final do tratamento é um dado importante se analisado sob a ótica da transferência, uma vez que se entende que as situações da vida atual figuram, em aspectos conflitivos, como uma reedição das situações passadas (Castro, 2020; Luborsky \& Crits-Christoph, 1990). Além do mais, a menção sobre a situação atual do paciente é comum de se ocorrer em psicoterapia (Luz, 2015). Espera-se que ao final da psicoterapia haja menor influência da transferência e maior sobre o relacionamento real e a realidade externa, pois esses elementos contribuem para uma atitude alicerçada no principio da realidade (Mondardo et al., 2009).

Por fim, pode-se entender que, em termos relacionais, oriundos das abordagens psicodinâmicas e numa tentativa de sumarizar os resultados desta pesquisa com os encontrados também nos estudos anteriores (Crits-Christoph et al., 2004; Duarte et al., 2001; Silva et al., 2013), os conflitos destes pacientes estão relacionados com a necessidade de um vínculo seguro de apego, de segurança e de amor com pessoas de referência, mas que, ao invés disto, houve a de falta de cuidado e amparo. Assim, emergiram sintomas emocionais relacionados com o desamparo, rejeição, abandono e falta de confiança (CritsChristoph et al., 2004), que gerariam possíveis falhas narcísicas e de simbolização. Destaca-se também que esse pressuposto é encontrado na associação entre ansiedade e o apego inseguro (Álvarez et al., 2011; Benetti et al., 2018; Crits-Christoph et al., 2004).

\section{Considerações Finais}

Neste estudo, constatou-se mudanças no conflito relacional central expressos entre a comparação do início com o final do tratamento de pacientes com sintomas de ansiedade em psicoterapia psicodinâmica on-line. Foi possível identificar que a modalidade de intervenção on-line ofertada foi efetiva para proporcionar mudanças nos relacionamentos interpessoais conflituosos e a promoção de atitudes psicológicas mais flexíveis pelas pacientes. Os resultados encontrados sinalizaram características que podem ser gerais de pacientes que apresentam sintomatologia ansiosa, uma vez que os achados ora salientados também foram corroborados com outros estudos já presentes na literatura em pacientes atendidos presencialmente.

Por se tratar de um estudo com um grupo pequeno e com a seleção de terapeutas e pacientes por conveniência, a generalização dos resultados é restrita e requer estudos subsequentes que possam ofertar replicação. Entretanto, os achados empíricos podem ajudar na compreensão da dinâmica relacional de pacientes ansiosos e o potencial de mudança intrapsíquica das intervenções psicodinâmicas no contexto a distância, possibilitando que os profissionais da área clínica proponham intervenções psicodinâmicas, na modalidade on-line, mais eficazes. Sugere-se que haja investimento em estudos que possam descrever as características dos processos psicoterápicos on-line, especialmente na abordagem psicodinâmica.

\section{Agradecimentos}

O presente trabalho foi realizado com apoio da Coordenação de Aperfeiçoamento de Pessoal de Nível Superior Brasil (CAPES) - Código de Financiamento 001.

\section{Referências}

Álvarez, M. P. S., González, M. G., \& Rojas, L. G. (2011). Apego y Psicopatología: Estudio comparativo de los estilos de apego en adultos con y sin sintomatología ansioso-depresiva. Revista Argentina de 20 (1), http://repositorio.ucm.cl/bitstream/handle/ucm/1733/garrido_1_apego_y.pdf?sequence=1\&isAllowed=y

Barak, A., Hen, L., Boniel-Nissim, M., \& Shapira, N. (2008). A Comprehensive Review and a Meta-Analysis of the Effectiveness of Internet- Based Psychotherapeutic Interventions. Journal of Technology in Human Services, 26(2/4), 109-160. https://doi.org/10.1080/15228830802094429 
Barrett, M. S. (2009). The Core Conflictual Relationship Theme: A Psychodynamic Formulation of the Case of Mrs Lewis. In P. Sturmey (Org.), Clinical case formulation: Varieties of approaches (p. 346). Wiley-Blackwell.

Benetti, S. P. C., Both, L., \& Feijo, L. P. (2018). A parentalidade no desenvolvimento da criança e do adolescente: contribuições psicanalítica. In L. F. Pessôa, D. M. L. F. Mendes, \& M. L. Seidel-de-moura (Orgs.), Parentalidade: Diferentes Perspectivas, Evidências e Experiências (p. 207). Appris Editora.

Beutel, M. E., Böhme, K., Banerjee, M., \& Zwerenz, R. (2018). Psychodynamic online treatment following supportive expressive therapy (SET):Therapeutic rationale, interventions and treatment process. Zeitschrift fur Psychosomatische Medizin und Psychotherapie, 64(2), 186-197. https://doi.org/10.13109/zptm.2018.64.2.186

Bottino, S. M. B., Junqueira, C., Bairrão, J. F. M. H., Hanns, L. A., Rosa, M. D., \& Andrade, L. H. S. G. (2003). Transtornos da compulsão alimentar periódica e psicoterapia: É possível sistematizar a formulação psicodinâmica de caso? Revista Brasileira de Psiquiatria, 25(3), 166-170. https://doi.org/10.1590/s151644462003000300009

Castro, F. C. A. (2020). Avaliação das narrativas interpessoais de uma paciente borderline em psicoterapia psicodinâmica. Universidade do Vale do Rio dos Sinos.

Creswell, J. W. (2010). Projeto de pesquisa: Métodos qualitativos, quantitativos e mistos ( $3^{\circ}$ ed). Artmed.

Crits-Christoph, P., Connolly, M. B., Azarian, K., Crits-Christoph, K., \& Shappell, S. (1996). An open trial of brief supportive-expressive psychotherapy in the treatment of generalized anxiety disorder. Psychotherapy, 33(3), 418-430. https://doi.org/10.1037/0033-3204.33.3.418

Crits-Christoph, P., Gibbons, M. B. C., \& Crits-Christoph, K. (2004). Supportive-Expressive Psychodynamic Therapy. In R. G. Heimberg, C. L. Turk, \& D. S. Mennin (Orgs.), Generalized anxiety disorder: Advances in research and practice (p. 465). The Guilford Press.

Duarte, C. E., Cheniaux Júnior, E., Almeida, Y. A., Almeida, C. de P., Souza, F., Vieira, I. M. M. M., Arcoverde, M. A., Nunes, V. P., Zapata, M. R., \& Zusman, S. (2001). A verificação de temas centrais de conflito de relacionamento interpessoal em pacientes com transtornos de ansiedade: resultados preliminares. Revista Brasileira de Psicoterapia2, 3(2), 117-129.

Edwards, D. J. A. A. (2007). Collaborative Versus Adversarial Stances in Scientific Discourse: Implications for the Role of Systematic Case Studies in the Development of Evidence-Based Practice in Psychotherapy. Pragmatic Case Studies in Psychotherapy, 3(1), 6-34. https://doi.org/10.14713/pcsp.v3i1.892

Evans, C., Connell, J., Barkham, M., Marginson, F., McGrath, G., Mellor-Clark, J., \& Audin, K. (2002). Towards a standardised brief outcome measure: Psychometric properties and utility of the CORE-OM. The British Journal of Psychiatry, 180(1), 51-60. https://doi.org/https://doi.org/10.1192/bjp.180.1.51

Feijó, L. P., Fermann, I. L., Andretta, I., \& Serralta, F. B. (2018). Eficácia de tratamentos psicoterápicos utilizando a internet: Revisão sistemática. Anais da $48^{a}$ Reunião Anual da Sociedade Brasileira de Psicologia.

Fernandes, M. A., Silva, D. R. A., Ibiapina, A. R. de S., \& Silva, J. S. (2018). Mental illness and its relationship with work: A study of workers with mental disorders. Revista Brasileira de Medicina do Trabalho, 16(3), 277-286. https://doi.org/10.5327/Z1679443520180110

Gaston, L., Piper, W. E., Debbane, E. G., Bienvenu, J. P., \& Gar Ant, J. (1994). Alliance and technique for predicting outcome in short-and long-term analytic psychotherapy. Psychotherapy Research, 4(2), 121-135. https://doi.org/10.1080/10503309412331333952

Jacobson, N. S., \& Truax, P. (1991). Clinical Significance: A Statistical Approach to Defining Meaningful Change in Psychotherapy Research. Journal of Consulting and Clinical Psychology, 59(1), 12-19. https://doi.org/10.1037/0022-006X.59.1.12

López Moreno, C. M., Schalayeff, C., Acosta, S. R., Vernengo, P., Roussos, A. J., \& Lerner, B. D. (2005). Evaluation of psychic change through the application of empirical and clinical techniques for a 2-year treatment: A single case study. Psychotherapy Research, 15(3), 199-209. https://doi.org/10.1080/10503300512331387799

Luborsky, L. (1984). Principles of Psychoanalytic Psychotherapy. A Manual for Supportive-Expressive Treatment. Basic Books.

Luborsky, L., \& Crits-Christoph, P. (1990). Understanding transference: The Core Conflictual Relationship Theme method. In Understanding transference: The Core Conflictual Relationship Theme method (2nd ed.). Basic Books. https://doi.org/10.1037/10250-000

Luborsky, L., Popp, C., Luborsky, E., \& Mark, D. (1994). The Core Conflictual Relationship Theme. Psychotherapy Research, 4(3-4), 172-183. https://doi.org/10.1080/10503309412331334012

Luz, A. B. (2015). Fases da psicoterapia. In C. L. Eizirik, R. W. de Aguiar, \& S. Schestatsky (Orgs.), Psicoterapia de Orientação Analítica: Fundamentos Teóricos e Clínicos (p. 855). Artmed.

Malone, J. C. (2018). Relational psychoanalysis: Not a theory but a framework. In Introduction to Contemporary Psychoanalysis: Defining Terms and Building Bridges (p. 208-226). https://doi.org/10.4324/9781315180120

Mondardo, A. H., Piovesan, L., \& Mantovani, P. C. (2009). A percepção do paciente quanto A percepção do paciente quanto ao processo de mudança psicoterápica. Aletheia, 30, 158-171.

Moreno, A. L., DeSousa, D. A., Souza, A. M. F. L. P., Manfro, G. G., Salum, G. A., Koller, S. H., Osório, F. L., \& Crippa, J. A. S. (2016). Factor Structure, Reliability, and Item Parameters of the Brazilian-Portuguese Version of the GAD-7 Questionnaire. Temas em Psicologia, 24(1), 367-376. https://doi.org/10.9788/TP2016.1-25

Rees, C. S., \& Maclaine, E. (2015). A Systematic Review of Videoconference-Delivered Psychological Treatment for Anxiety Disorders. Australian Psychologist, 50(4), 259-264. https://doi.org/10.1111/ap.12122 
Research, Society and Development, v. 11, n. 2, e41711225918, 2022

(CC BY 4.0) | ISSN 2525-3409 | DOI: http://dx.doi.org/10.33448/rsd-v11i2.25918

Rocha, G. M. A. (2013). O método do tema central do relacionamento conflituoso - CCRT. In E. M. P. Yoshida \& M. L. E. Enéas (Orgs.), Psicoterapias psicodinâmicas breves: propostas atuais. Editora Alínea.

Rosbrow, T. (1995). Review of Understanding transference: The CCRT method. Psychoanalytic Psychology, 12(4), 607-610. https://doi.org/10.1037/h0085203

Salgado, A. I. M., \& Pires, A. A. P. (2014). Avaliação da mudança nas relações interpessoais através do CCRT: Estudo de caso psicanalítico. Psicologia Clínica, 26(1), 135-150. http://www.redalyc.org/articulo.oa?id=291031730009

Santos, A. (2013). O impacto do CCRT na avaliação e diagnóstico em psicoterapia. Revista Iberoamericana de Diagnostico y Evaluacion Psicologica, 1(35), 113-137. http://www.scopus.com/inward/record.url?eid=2-s2.0-84902456933\&partnerID=tZOtx3y1

Serralta, F. B., Nunes, M. L. T., \& Eizirik, C. L. (2011). Considerações metodológicas sobre o estudo de caso na pesquisa em psicoterapia. Estudos de Psicologia (Campinas), 28(4), 501-510. https://doi.org/10.1590/S0103-166X2011000400010

Shedler, J. (2010). The Efficacy of Psychodynamic Psychotherapy. American Psychologist, 65(2), 98-109. https://doi.org/10.1037/a0018378

Silva, T. A. H., \& Serralta, F. B. (2013). Padrão de conflito nos relacionamentos: Um estudo de caso através do método CCRT (p. 1). https://lume.ufrgs.br/bitstream/handle/10183/91417/Poster_29215.pdf?sequence=2\&isAllowed=y

Spitzer, R. L., Kroenke, K., Williams, J. B. W., \& Löwe, B. (2006). A brief measure for assessing generalized anxiety disorder: The GAD-7. Archives of Internal Medicine, 166(10), 1092-1097. https://doi.org/10.1001/archinte.166.10.1092

Sturza, J. M., \& Marques, A. D. (2017). A Importância do Trabalho para a Consolidação da Dignidade do Homem: Apontamentos sob a Perspectiva dos Direitos Sociais. Revista Direito, Estado e Sociedade, 50, 109-125. https://doi.org/10.17808/des.50.448

Varker, T., Brand, R. M., Ward, J., Terhaag, S., \& Phelps, A. (2018). Efficacy of Synchronous Telepsychology Interventions for People With Anxiety, Depression, Posttraumatic Stress Disorder, and Adjustment Disorder: A Rapid Evidence Assessment. Psychological Services. https://doi.org/10.1037/ser0000239

Wilczek, A., Weinryb, R. M., Barber, J. P., Gustavsson, J. P., \& Åsberg, M. (2004). Change in the core conflictual relationship theme after long-term dynamic psychotherapy. Psychotherapy Research, 14(1), 107-125. https://doi.org/10.1093/ptr/kph007

Yoshida, E. M. P., Eliseu, S., Silva, F. R. C. S. S., Finotelli, I., Sanches, F. M., Penteado, E. F., Massei, A. C., Rocha, G. M. A., \& Enéas, M. L. E. (2009). Psicoterapia psicodinâmica breve: estratégia terapêutica e mudança no padrão de relacionamento conflituoso. Psico USF, 14(3), 275-285. https://doi.org/10.1590/S1413-82712009000300004

Zwerenz, R., Becker, J., Gerzymisch, K., Siepmann, M., Holme, M., Kiwus, U., Spörl-Dönch, S., \& Beutel, M. E. (2017). Evaluation of a transdiagnostic psychodynamic online intervention to support return to work: A randomized controlled trial. PLoS ONE, 12(5). https://doi.org/10.1371/journal.pone.0176513 